\title{
Direct monitoring of plasmon resonances in a tip-surface gap of varying width
}

\author{
Bruno Pettinger, ${ }^{1}, *$ Katrin F. Domke, ${ }^{1}$ Dai Zhang, ${ }^{1}$ Rolf Schuster, ${ }^{2}$ and Gerhard Ertl ${ }^{1}$ \\ ${ }^{1}$ Fritz-Haber-Institut der Max-Planck-Gesellschaft, Faradayweg 4-6, D-14195 Berlin, Germany \\ ${ }^{2}$ Institut für Physikalische Chemie, Universität Karlsruhe, D-76128 Karlsruhe, Germany
}

(Received 8 June 2007; published 21 September 2007)

\begin{abstract}
We have studied the dependence of tip-enhanced Raman (TER) spectra composed of discrete Raman lines and an intense Lorentzian-shaped background on varying interparticle distance. For increasing tip-sample separation, we observe a remarkable blueshift of the background maximum that is ascribed to an energy shift of the localized surface plasmon resonance. In addition, we report on a steep background and Raman band intensity decrease within 5-10 nm tip retraction that highlights the underlying near-field enhancement mechanism of TER spectroscopy.
\end{abstract}

DOI: 10.1103/PhysRevB.76.113409

PACS number(s): 68.37.Uv

The wide applicability of plasmonics in nanoscale spectroscopy ${ }^{1}$ and lithography, ${ }^{2}$ biosensors, ${ }^{3,4}$ and subwavelength photonic devices ${ }^{5}$ shows the importance of this emerging field for chemists, physicists, and materials scientists alike. The excitation of localized surface plasmons (LSPs) in metal nanostructures enables confinement of (visible) light to the nanometer length scale. LSPs mediate enhanced excitation and radiation processes of molecules located in the LSP near-field zone. The LSP resonances strongly depend on the excitation wavelength, the particle material, dimension, geometry, as well as interparticle distances. ${ }^{6}$ These effects play a crucial role in spectroscopies based on electromagnetic field enhancement processes, ${ }^{1,7-19}$ but are difficult to address experimentally.

A tip-metal cavity, as present in the tip-enhanced Raman spectroscopy (TERS) experiment, represents a quite simple, but efficient photonic unit with a tunable gap length. In this paper, we present a complete analysis of the TER response composed of discrete Raman lines and the spectral background (bg) (which is usually neglected, though it exhibits in general much more integral intensity than all the discrete Raman lines together). With the latter, we provide precise experimental evidence for the theoretically predicted dependence of the LSP energies on the interparticle distance in the few-nanometer range. In addition, we show that the intensities of the spectral background and of the Raman bands exhibit the same decay profile with increasing gap width up to $z<8 \mathrm{~nm}$. This has an important consequence for supporting or ruling out mechanisms for the spectral bg.

Let us conceptualize the enhancement as a process involving three major steps which are summarized in Eqs. (1)-(3) (we neglect here rather minor paths of light scattering such as a direct scattering of a LSP into a photon). First, an incoming photon $\left(\hbar \omega_{i}\right)$ excites a LSP of corresponding energy. In the case discussed here, the LSP resonance is determined by the geometrical and electrodynamical properties of the tip-substrate cavity ${ }^{20}$ and is therefore also called gap mode. ${ }^{21}$ In our notation, the nonexcited and excited tip-adsorbatesubstrate cavities are symbolized by (TAS)[0] and (TAS) $\left[\mathrm{LSP}_{\hbar \omega}\right]$, respectively, with $\mathrm{LSP}_{\hbar \omega}$ indicating the excitation of LSP of energy $\hbar \omega$.

The second step [Eq. (2)] describes the (virtual) excitation and deexcitation of $A$ involving the annihilation of $\operatorname{LSP}_{\hbar \omega_{i}}$ and the creation of $\operatorname{LSP}_{\hbar \omega_{x}}$ with energy $\hbar \omega_{x}$. $A^{*}$ denotes the adsorbate in an electronically excited state. Step (2) includes both the inelastic scattering of LSPs leading to the discrete Raman lines $(x=$ TERS $)$ indicative of the adsorbate vibrational signature, and the electronic excitation and deexcitation of the adsorbate-substrate complex, seen as bg in the spectra $(x=b g)$.

In step (3) the inelastically scattered $\mathrm{LSP}_{\hbar \omega_{x}}$ become annihilated by the emission of a photon of energy $\hbar \omega_{x}$.

$$
\begin{gathered}
\hbar \omega_{i}+(\mathrm{TAS})[0] \rightarrow(\mathrm{TAS})\left[\operatorname{LSP}\left(\hbar \omega_{i}\right)\right] \\
(\mathrm{TAS})\left[\operatorname{LSP}\left(\hbar \omega_{i}\right)\right] \rightarrow\left(\mathrm{TA}^{*} \mathrm{~S}\right)[0] \rightarrow(\mathrm{TAS})\left[\operatorname{LSP}\left(\hbar \omega_{x}\right)\right]
\end{gathered}
$$

$$
(\mathrm{TAS})\left[\operatorname{LSP}\left(\hbar \omega_{x}\right)\right] \rightarrow(\mathrm{TAS})[0]+\hbar \omega_{x} .
$$

From a quantum mechanical viewpoint, scattering processes usually occur in such a short time frame that all permutations of the sequence of excitation and annihilation processes should be considered (and not only the sequence described above). ${ }^{22}$

A detailed description of TERS principles and experimental setup is given elsewhere. ${ }^{1,16-18,24}$ The resonant cavity is formed by a conical $\mathrm{Au}$ scanning tunneling microscopy (STM) tip of $20 \mathrm{~nm}$ radius located at a piezocontrolled distance $z$ above an atomically smooth $\mathrm{Au}(111)$ substrate. An adsorbate layer is sandwiched between the STM tip and the metal substrate. It is exposed to the strong electromagnetic near-field created underneath the tip, giving rise to TER scattering composed of a spectrally broad background and discrete Raman bands. The experiments are performed with a monolayer of guanine and perchlorate coadsorbed on a $\mathrm{Au}(111)$ single crystal surface, previously prepared by flame annealing. ${ }^{23}$ Coadsorption with guanine leads to a stable perchlorate coverage at the $\mathrm{Au}(111)$ surface. A He-Ne laser beam $(632.8 \mathrm{~nm}, 0.5 \mathrm{~mW}, p$-polarized $)$ is focused to a spot of about $2 \mu \mathrm{m}$ diameter and directed onto the STM tip apex. During tunneling (constant-current mode at $1 \mathrm{nA}$ and $150 \mathrm{mV}$ bias), the tip rests at about $1 \mathrm{~nm}$ distance above the sample surface.

To measure the tip-sample distance dependence of the TER signal, the feedback loop of the STM is gated and the $z$ 


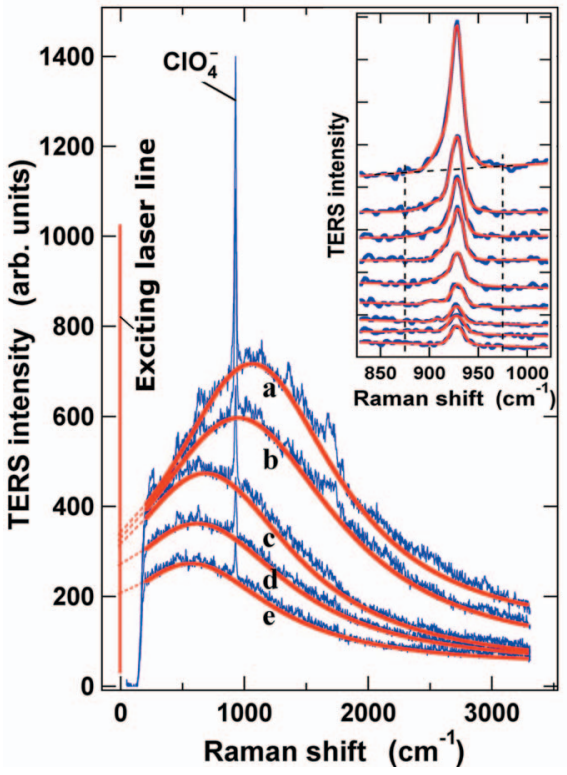

FIG. 1. (Color) TER spectra for coadsorbed guanine and $\mathrm{ClO}_{4}^{-}$ for different distances $z$ of the STM tip to the surface: Five spectra from a series of 15; (a)-(e) $z=1-5.2 \mathrm{~nm}$. Retraction speed: $0.16 \mathrm{~nm} / \mathrm{s}$. Smooth red lines: bg curves from a Lorentzian fit. Inset: set of nine spectra of the same series and their fit curves (red curves) expanded around $930 \mathrm{~cm}^{-1}$.

position of the tip is controlled by a programmable ramp generator. Tip retraction with constant speed is started $5-10 \mathrm{~s}$ after gating the feedback loop. From the small intensity fluctuations of the TER signal during the initial holding time without tip retraction, the accuracy of the actual tip position is estimated to be about $\pm 0.3 \mathrm{~nm}$ during gating the feedback loop. While retracting the STM tip, a series of Raman spectra ( $1 \mathrm{~s}$ integration time) is taken at intervals of about $1.5 \mathrm{~s}$. The bg of the obtained TER spectra and the Raman lines are fitted with Lorentzian and with Gaussian curves, respectively, to facilitate their analysis (Fig. 1).

The recorded spectra show Raman bands of guanine and perchlorate on top of an intense, Lorentzian-shaped bg (Fig. 1). We will address the bg and its spectral changes upon increasing the metal-tip substrate distance $z$ before we discuss the corresponding intensity changes of the Raman lines. Nonenhanced Raman spectra usually exhibit sharp lines over flat spectral characteristics with very weak intensities. In contrast, enhanced Raman spectra display a large bg underneath the Raman lines, although they are often presented after bg correction in the literature. It is worthwhile to take a closer look at the bg for once, as it contains by far more integral intensity than the sum of the discrete Raman lines. In general, the bg represents inelastic light scattering from the adsorbate-substrate system. ${ }^{19,25-28,30,31}$

At $z=1 \mathrm{~nm}$, the bg maximum is located at a Raman shift of about $1075 \mathrm{~cm}^{-1}$ and has a rather narrow halfwidth of about $650 \mathrm{~cm}^{-1}$. The Raman shift is referenced to the frequency of the excitation laser of $15802.8 \mathrm{~cm}^{-1}$. On an absolute scale, the frequency of the bg maximum corresponds to about $14727.8 \mathrm{~cm}^{-1}$, i.e., to a photon energy of $1.826 \mathrm{eV}$. The full width at half maximum (FWHM) of the bg corre-

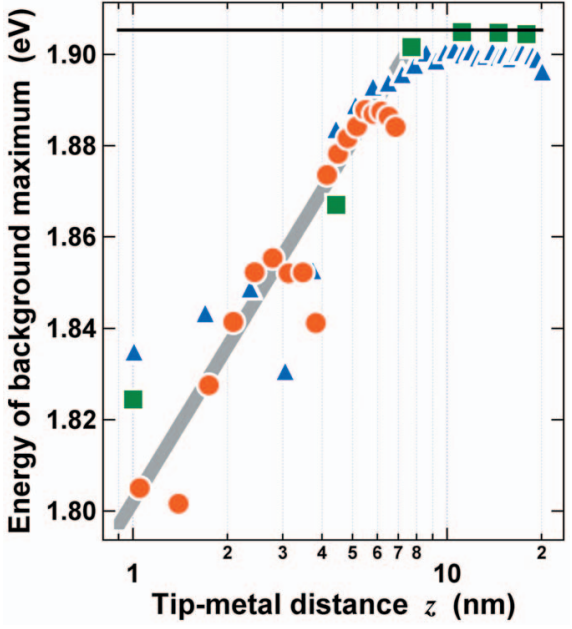

FIG. 2. (Color) Plot of the energy of the bg maximum vs tipmetal distance. The solid gray line is a guide to the eye. Retraction speeds: $(\boldsymbol{\square}) 1.6 \mathrm{~nm} / \mathrm{s},(\boldsymbol{\Delta}) 0.32 \mathrm{~nm} / \mathrm{s}$, and $(\bullet) 0.16 \mathrm{~nm} / \mathrm{s}$. Horizontal line: energy of the bg maximum of a free tip at $1.906 \mathrm{eV}$.

sponds to about $0.08 \mathrm{eV}$. Upon retraction of the tip, the intensity of the bg strongly decreases with increasing $z$ [Figs. $1(b)-1(e)$ ]. In addition, there is a remarkable shift of the energy of the bg maximum toward higher energy with increasing tip-surface separation. In Fig. 1, the energy shifts from about $1.826 \mathrm{eV}$ for $z=1 \mathrm{~nm}$ to about $1.882 \mathrm{eV}$ for $z$ $=5.2 \mathrm{~nm}$. It is noteworthy that the FWHM of the bg remains about constant during tip retraction. As discussed later, similar shifts were observed in other experiments with different tip-retraction speeds and increased total retraction distances up to $70 \mathrm{~nm}$. An isolated illuminated tip $(z \rightarrow \infty)$ shows a weak bg centered at $1.906 \mathrm{eV}$. In Fig. 2, the strong energy shift of the bg maximum is plotted as a function of the tipsurface distance up to $20 \mathrm{~nm}$ for three series of Raman spectra.

Itoh et al. showed for colloidal aggregates that the energy maximum of the bg in enhanced Raman spectra coincides with the individual resonance energy of the LSP of these systems. ${ }^{28}$ Translating this observation to our situation, we propose that initially the bg profile is rather flat, ${ }^{29}$ but now displays the mode structure and energy profiles of LSPs that are excited in the tip-metal cavity. ${ }^{21}$ Hence, the bg becomes Lorentzian shaped like the LSP resonance. By monitoring the bg maximum shift with varying tip-sample distance, we can directly observe the corresponding changes in the gap resonance.

The spectral blueshift of the bg must be ascribed to a blueshift of LSP energies due to a decreasing electrodynamic coupling between the excited surface plasmons of the tip and the gold substrate with increasing tip-surface distance. The concept of LSPs was introduced by Rendell et al. who calculated the LSP modes for a metal sphere $/ \mathrm{Al}_{2} \mathrm{O}_{3} / \mathrm{Al}$ substrate system dependent on the sphere diameter and the $\mathrm{Al}_{2} \mathrm{O}_{3}$ thickness. ${ }^{20}$ Indeed, a blueshift of the LSP frequencies was predicted for increasing sphere-substrate distances. Similar blueshifts were reported in more recent theoretical papers. ${ }^{32-35}$ Porto et al. ${ }^{35}$ calculated LSP energies for a gold 
sphere of $40 \mathrm{~nm}$ radius above a $\mathrm{Au}$ substrate of $1.82,1.87$, and $2.03 \mathrm{eV}$ at sphere-sample distances of 1,2 , and $5 \mathrm{~nm}$. Taking into account the differences in radius and shape of our tip $(R=20 \mathrm{~nm})$ compared to the sphere used in the calculations of Porto et al. $(R=40 \mathrm{~nm})$, the values of the LSP energies obtained by theory are remarkably close to our observed maximum energies. Important is that both the excitation and the Raman lines are located well within the observed LSP resonance of a FWHM of $0.08 \mathrm{eV}$ (see Fig. 1).

At larger distances above 5-8 nm, the experimental maximum frequency levels off and stays about constant for $z>10 \mathrm{~nm}$. This indicates that the mutual influence of tip and surface ceases rather abruptly at about $8 \mathrm{~nm}$ separation. The observed limit frequency at large distances should therefore represent the LSP of an isolated particle. Indeed, the observed value of approximately $1.906 \mathrm{eV}$ agrees very well with the maximum energy of the bg measured for our isolated tip (Fig. 2). In addition, this value is in reasonable agreement with the surface plasmon frequency calculated for an ellipsoidal $\mathrm{Au}$ particle of dimensions of the tip apex. ${ }^{36} \mathrm{In}$ this context please note that energy shifts were reported, for example, for lithographically prepared particle pairs but for a distance range of $\sim 10-100 \mathrm{~nm}$ and an accuracy of only $10 \mathrm{~nm} .{ }^{37}$ In our experiments, we observe a particular strong near-field coupling for distances between 1 and $10 \mathrm{~nm}$.

In contrast to the frequency of the bg maximum, the frequencies of the discrete Raman lines, indicative of the adsorbate vibrational frequencies, do not change with tip-substrate distance (Fig. 1, inset). However, similarly to the bg, the intensities of the Raman lines also strongly decrease with increasing tip-substrate separation. The integrated intensities of both the bg and of a characteristic, intense Raman band at $930 \mathrm{~cm}^{-1}$ are plotted vs the tip-surface distance in Fig. 3 . The coadsorption of guanine and perchlorate gives weak guanine bands and three, more intense perchlorate bands. The most intense band at $930 \mathrm{~cm}^{-1}$ is assigned to the $A_{1}$ breathing mode of perchlorate (Fig. 1, curve a). In contrast to the guanine bands which decrease slowly with proceeding illumination due to bleaching, the $\mathrm{ClO}_{4}^{-}$band intensity remains unaffected. Therefore, for quantitative Raman intensity vs $z$ profiles, the intensity of this band was evaluated. It should be emphasized that the intensities of guanine bands exhibit similar behavior with increasing $z$.

The data are extracted from three series of TER spectra with total retraction lengths of 6,12 , and $50 \mathrm{~nm}$ (retraction speeds of $0.16,0.32$, and $1.6 \mathrm{~nm} / \mathrm{s}$, respectively). The integral intensities were normalized to 1 at $z=1 \mathrm{~nm}$, which is the approximate tip-surface distance in tunneling contact.

The bg intensity [Fig. 3(a)] shows a rapid decay within the first $8 \mathrm{~nm}$, followed by a slower decrease that is nearly linear in this double logarithmic scale (varying from about $7 \%$ of the initial signal at $z=8 \mathrm{~nm}$ to about $3 \%$ at $z=70 \mathrm{~nm}$, not shown). The TER band intensities exhibit the same steep intensity decrease with increasing tip-surface distance, reaching about one-hundredth of the initial intensity at $z \approx 20 \mathrm{~nm}$ tip-surface distance. For comparison, a fit curve to the Raman band data is presented as a solid gray line in Fig. 3(b). The same fit curve is also plotted in Fig. 3(a). Obviously, the Raman line and bg intensities exhibit the same tip-sample distance dependence up to $z \approx 8 \mathrm{~nm}$. The small contribution

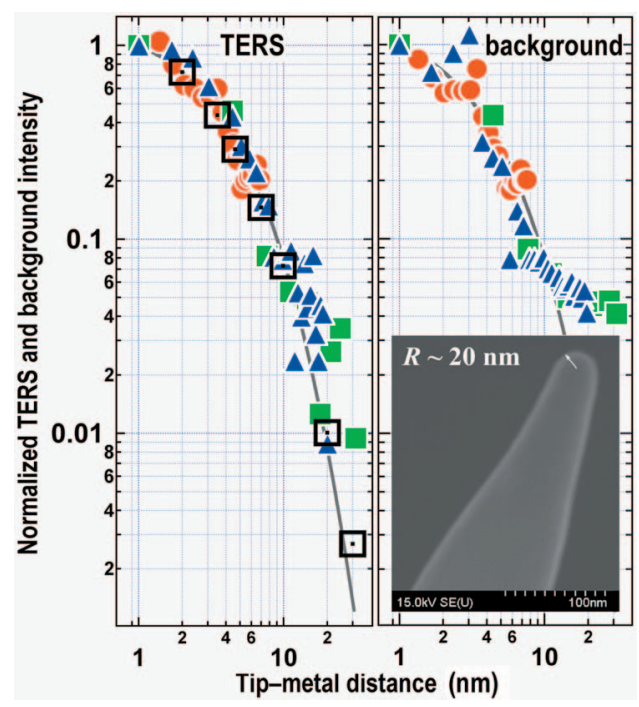

FIG. 3. (Color) Double logarithmic plot of the integral bg and Raman band intensities for varying distances $z$ of the STM tip to the surface in panels (a) and (b), respectively. Panel (a): inset shows the scanning electron microscope image of the STM tip. Panel (b): Raman intensities of the $\mathrm{ClO}_{4}^{-}$stretch are plotted vs $z$. Retraction

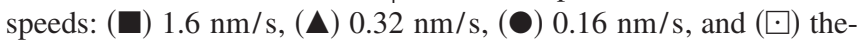
oretical data in panel (b) from Fig. 4(b) of Ref. 39. Gray lines in both panels: the same fit curve used as a guide to the eyes.

to the bg in the percentage range with a different $z$ dependence indicates an additional scattering process of hitherto unkown origin.

At this point it should be noted that our STM-TER intensity profile, involving a strong electrodynamic Au-tip Ausubstrate coupling, shows a significantly faster decay with increasing tip-surface distance $z$ than the atomic force microscopy-TER intensity profile reported by Hartschuh et al. (for a $15 \mathrm{~nm}$ radius $\mathrm{Ag}$ tip over a glassy carbon substrate).$^{38}$

In Fig. 3(b), we also included data from recent finite element calculations by Notingher et al. of the distance dependence of the Raman enhancement underneath a tip with $R$ $=20 \mathrm{~nm}$ in air [Fig. 4(b) of Ref. 39]. Since in those calculations the minimum tip-surface distance was $2 \mathrm{~nm}$, we normalized the theoretical intensities of Notingher et al. to our average value at $2 \mathrm{~nm}$ distance. Apparently, our experimental data match the calculations over 2 orders of magnitude. This is very surprising because in the calculations of Notingher $e t$ al. no substrate surface is taken into account and the Raman enhancement refers only to the position directly beneath the tip apex, not considering the lateral extent of the Raman enhancement.

In summary, we present the dependence of the TER spectra on the tip-substrate distance. We report on a remarkable blueshift of the bg maximum upon tip retraction that experimentally visualizes the long-since theoretically predicted blueshift of LSP energy with increasing interparticle distance over a range of more than $10 \mathrm{~nm}$. Also presented is a comparison of the tip-sample distance dependence of the bg and TER band intensities, showing the same rapid decay (for $z$ $<8 \mathrm{~nm}$ ). In our view, this points to an at least similar under- 
lying near-field enhancement mechanism for both kind of inelastic scattering. The fast intensity decay illustrates the high vertical spatial resolution of TERS.
D.Z. gratefully acknowledges the support by the Max Planck Society. Support by the Fonds der Chemischen Industrie is gratefully acknowledged by R.S. *pettinger@fhi-berlin.mpg.de

${ }^{1}$ B. Pettinger, B. Ren, G. Picardi, R. Schuster, and G. Ertl, Phys. Rev. Lett. 92, 096101 (2004).

${ }^{2}$ K. Salaita, Y. Wang, J. Fragala, R. A. Vega, C. Liu, and C. A. Mirkin, Angew. Chem., Int. Ed. 45, 7220 (2006).

${ }^{3}$ A. P. F. Turner, Science 290, 1315 (2000).

${ }^{4}$ A. R. Mendelsohn and R. Brent, Science 284, 1948 (1999).

${ }^{5}$ E. Ozbay, Science 311, 1 (2006).

${ }^{6}$ T. A. Kelf, Y. Sugawara, J. J. Baumberg, M. Abdelsalam, and P. N. Bartlett, Phys. Rev. Lett. 95, 116802 (2005).

${ }^{7}$ R. M. Stöckle, Y. D. Suh, V. Deckert, and R. Zenobi, Chem. Phys. Lett. 318, 131 (2000).

${ }^{8}$ M. S. Anderson, Appl. Phys. Lett. 76, 3130 (2000).

${ }^{9}$ B. Pettinger, G. Picardi, R. Schuster, and G. Ertl, Electrochemistry (Tokyo, Jpn.) 68, 942 (2000).

${ }^{10}$ L. T. Nieman, G. M. Krampert, and R. E. Martinez, Rev. Sci. Instrum. 72, 1691 (2001).

${ }^{11}$ N. Hayazawa, Y. Inouye, Z. Sekhat, and S. Kawata, J. Chem. Phys. 117, 1296 (2002).

${ }^{12}$ B. Pettinger, G. Picardi, R. Schuster, and G. Ertl, Single Mol. 3, 285 (2002)

${ }^{13}$ N. Hayazawa, T. Yano, H. Watanabe, Y. Inouye, and S. Kawata, Chem. Phys. Lett. 376, 174 (2003).

${ }^{14}$ M. Micic, N. Klymyshyn, Y. D. Suh, and H. P. Lu, J. Phys. Chem. B 107, 1574 (2003).

${ }^{15}$ D. Hu, M. Micic, N. Klymyshyn, Y. D. Suh, and H. P. Lu, Rev. Sci. Instrum. 74, 3347 (2003).

${ }^{16}$ B. Pettinger, G. Picardi, R. Schuster, and G. Ertl, J. Electroanal. Chem. 554, 293 (2003).

${ }^{17}$ B. Pettinger, B. Ren, G. Picardi, R. Schuster, and G. Ertl, J. Raman Spectrosc. 36, 541 (2005).

${ }^{18}$ B. Ren, G. Picardi, B. Pettinger, R. Schuster, and G. Ertl, Angew. Chem., Int. Ed. 44, 139 (2005).

${ }^{19}$ K. F. Domke, D. Zhang, and B. Pettinger, J. Am. Chem. Soc. 128, 14721 (2006).

${ }^{20}$ R. W. Rendell, D. J. Scal, and B. Mühlschlegel, Phys. Rev. Lett. 41, 1746 (1978).

${ }^{21}$ S. Hayashi, Top. Appl. Phys. 81, 71 (2001).
${ }^{22}$ The Quantum Theory of Light, edited by R. Loudon (Oxford Science, Oxford, 1980).

${ }^{23}$ J. Clavilier, R. Faure, G. Guinet, and R. Durand, J. Electroanal. Chem. Interfacial Electrochem. 107, 205 (1980).

${ }^{24}$ B. Ren, G. Picardi, and B. Pettinger, Rev. Sci. Instrum. 75, 837 (2004).

${ }^{25}$ J. Jiang, B. Bosnick, M. Maillard, and L. Brus, J. Phys. Chem. B 107, 9964 (2003).

${ }^{26}$ T. Itoh, J. Biju, M. Ishikawa, Y. Kikkawa, K. Hashimoto, A. Ikehata, and Y. Ozaki, J. Chem. Phys. 124, 134708 (2006).

${ }^{27}$ T. Itoh, K. Hashimoto, J. Biju, M. Ishikawa, B. R. Wood, and Y. Ozaki, J. Phys. Chem. B 110, 9579 (2006).

${ }^{28}$ T. Itoh, Y. Kikkawa, J. Biju, M. Ishikawa, A. Ikehata, and Y. Ozaki, J. Phys. Chem. B 110, 21536 (2006).

${ }^{29}$ The nearly complete fluorescence quenching for species adsorbed at metal surfaces leads to a substantially reduced lifetime of the excited state. Therefore, if the bg originates from fluorescence, the remaining optical transitions would exhibit a broadened spectral band that appears rather flat in an energy interval of a few tens of eV. Yet, these transitions will be exposed to a wavelength dependent enhancement as other optical processes occurring in the near-field zone.

${ }^{30}$ A. Weiss and G. Haran, J. Phys. Chem. B 105, 12348 (2001).

${ }^{31}$ A. A. Moore, M. L. Jacobson, N. Belabas, K. L. Rowlen, and D. M. Jonas, J. Am. Chem. Soc. 127, 7292 (2005).

${ }^{32}$ D. L. Mills, Phys. Rev. B 65, 125419 (2002).

${ }^{33}$ S. Wu and D. L. Mills, Phys. Rev. B 65, 205420 (2002).

${ }^{34}$ C. Girard, Appl. Opt. 31, 5380 (1994).

${ }^{35}$ J. A. Porto, P. Johansson, S. P. Apell, and T. Lopez-Rios, Phys. Rev. B 67, 085409 (2003).

${ }^{36}$ P. I. Geshev, S. Klein, T. Witting, K. Dickmann, and M. Hietschold, Phys. Rev. B 70, 075402 (2004).

${ }^{37}$ L. Gunnarson, T. Rindzevicius, J. Prikulis, B. Kasemo, M. Kll, S. Zou, and G. C. Schatz, J. Phys. Chem. B 109, 1079 (2005).

${ }^{38}$ A. Hartschuh, E. J. Sanchez, X. S. Xie, and L. Novotny, Phys. Rev. Lett. 90, 095503 (2003).

${ }^{39}$ I. Notingher and A. Elfick, J. Phys. Chem. B 109, 15699 (2005). 